\title{
A New Limit on the Flux of Cosmic Antihelium*
}

\author{
T. Saeki, ${ }^{1}$ K. Anraku, ${ }^{1}$ S. Orito,${ }^{1}$ J. Ormes,${ }^{5}$ M. Imori,${ }^{1}$ B. Kimbell, ${ }^{3}$ Y. Makida, ${ }^{2}$ H. Matsumoto, ${ }^{4}$ H. Matsunaga ${ }^{1}$ \\ J. Mitchell, ${ }^{5}$ M. Motoki, ${ }^{4}$ J. Nishimura,${ }^{6}$ M. Nozaki,${ }^{4}$ M. Otoba, ${ }^{1}$ T. Sanuki,${ }^{1}$ R. Streitmatter, ${ }^{5}$ J. Suzuki, ${ }^{2}$ \\ K. Tanaka, ${ }^{2}$ I. Ueda, ${ }^{1}$ N. Yajima,${ }^{6}$ T. Yamagami, ${ }^{6}$ A. Yamamoto, ${ }^{2}$ T. Yoshida ${ }^{2}$ and K. Yoshimura ${ }^{1}$ \\ ${ }^{1}$ University of Tokyo, Bunkyo-ku, Tokyo 113, Japan \\ ${ }^{2}$ High Energy Accelerator Research Organization (KEK), Tsukuba, Ibaraki 305, Japan \\ ${ }^{3}$ New Mexico State University, Las Cruces, NM 88003, U.S.A \\ ${ }^{4}$ Kobe University, Kobe, Hyogo 657, Japan \\ ${ }^{5}$ National Aeronautics and Space Administration, Goddard Space Flight Center (NASA/GSFC), Greenbelt, MD 20771, U.S.A. \\ ${ }^{6}$ The Institute of Space and Astronautical Science (ISAS), Sagamihara, Kanagawa 229, Japan
}

(January 22, 1998)

\begin{abstract}
A very sensitive search for cosmic-ray antihelium was performed using data obtained from three scientific flights of BESS magnetic rigidity spectrometer. We have not observed any antihelium; this places a model-independent upper limit (95\% C.L.) on the antihelium flux of $6 \times 10^{-4} \mathrm{~m}^{-2} \mathrm{sr}^{-1} \mathrm{~s}^{-1}$ at the top of the atmosphere in the rigidity region 1 to $16 \mathrm{GV}$, after correcting for the estimated interaction loss of antihelium in the air and in the instrument. The corresponding upper limit on the $\overline{\mathrm{He}} / \mathrm{He}$ flux ratio is $3.1 \times 10^{-6}, 30$ times more stringent than the limits obtained in similar rigidity regions with magnetic spectrometers previous to BESS.
\end{abstract}

PACS numbers: 98.80.Cq, 98.70.Sa, 98.80.Bp, 98.90.+s

Cosmic-ray observations provide most direct evidence for our Galaxy being composed mostly by baryons. This baryon-antibaryon asymmetry can be global in the Universe, being created in the very early Universe due to the violations of $\mathrm{CP}$ and of baryon-number. However, depending on the nature of $\mathrm{CP}$ violation, baryon-symmetric models are conceivable [1] in which the Universe is separated into an equal number of matter- and antimatterdomains. Whereas $\gamma$-ray observations place strong limitations on the antimatter in our Galaxy and in the local cluster of galaxies, the domain structure could still exist beyond this scale. Although antihelium might be in principle produced in cosmic-ray interactions, their contribution to the $\overline{\mathrm{He}} / \mathrm{He}$ flux ratio is expected to be much smaller than $10^{-12}[2]$. Detecting antihelium at a level higher than this could therefore provide the evidence of antimatter domains or of other exotic phenomena such as superconducting strings in our Galaxy [3]. For further discussion of astrophysical considerations regarding the search for antihelium see the references $[4,5]$. We report in this letter (for more detail see [6]) a sensitive search for antihelium using the data from the '93, '94, and '95 flights of BESS detector, and provide model-independent upper limits on the absolute antihelium flux as well as on the $\overline{\mathrm{He}} / \mathrm{He}$ flux ratio. A limit on the flux ratio from an early analysis of '95 data is published elsewhere [4].

Fig. 1 shows front- and side-views of the BESS '95 instrument. The cylindrical configuration provides a wide tracking region and an acceptance of up to $0.32 \mathrm{~m}^{2} \mathrm{sr}$ depending on the off-line fiducial cuts. From inside to outside, it includes a jet-type drift (JET) chamber, in-

* This work is dedicated to the memory of Dr. R. Golden. ner drift chambers (IDCs), a superconducting solenoid, outer drift chambers (ODCs), and a time of flight (TOF) hodoscope. The solenoid produces a magnetic field of 1 $\mathrm{T}$ with an uniformity of $\pm 15 \%$ inside the bore.

The JET chamber [7], as the key tracking detector, measures up to 24 points per track three-dimensionally, each with the resolution [8] of $200 \mu \mathrm{m}$ in $r \phi$ plane and of 2 $\mathrm{cm}$ in $z$-position (a cylindrical coordinate $(r \phi z)$ is determined by defining the magnetic field direction as $z$-axis). Each of the IDCs and ODCs consists of two 12-mmthick drift layers which are divided into 50 -mm-wide cells, and measures $r \phi$ position each with $200 \mu \mathrm{m}$ resolution. These redundant and continuous position measurements with ODCs, IDCs, and JET chamber, all equipped with multi-hit capacity, make it possible to recognize multitrack events and tracks having interactions and scatterings, thus minimizing the background originating from the interactions. The $r \phi$-tracking in the central region is performed by fitting hit points in the JET chamber as well as in the IDCs. This results in a maximum detectable rigidity $(R)$ of $200 \mathrm{GV}$ and a typical rigidity resolution of $0.5 \%$ at $1 \mathrm{GV}$. Using vernier pads with a cycle of $10 \mathrm{~cm}$ for IDCs and $12 \mathrm{~cm}$ for ODCs, both chambers can measure the $z$ position, modulo the cycle, with $300 \mu \mathrm{m}$ resolution. Combining the IDC and JET information, $z$-position of the track can be determined with a precision of $300 \mu \mathrm{m}$.

The TOF hodoscope, placed at a radius of $65 \mathrm{~cm}$, consists of eight upper and twelve lower plastic scintillators (Bicron 404), each of which has a dimension of $95 \mathrm{~cm}$ $\times 10 \mathrm{~cm} \times 2 \mathrm{~cm}$. The light signals from the scintillator are guided through acrylic light-guides and reach the photomultiplier-tubes (PMTs) attached on both ends. The timing and amplitude of the PMT signals are measured to determine the time of flight and $\mathrm{d} E / \mathrm{d} x$ of inci- 
dent particles with resolution of 110 ps and $10 \%$, respectively. For '93 and '94 instruments, the TOF hodoscope consisted of four upper and six lower plastic scintillators, each of which had a dimension of $110 \mathrm{~cm} \times 20 \mathrm{~cm} \times 2$ $\mathrm{cm}$. The timing and $\mathrm{d} E / \mathrm{d} x$ resolutions was $280 \mathrm{ps}$ and 15 $\%$, respectively. A detailed description of '93 instrument has been published elsewhere [9].

These detector-components along with electronics are enclosed by a 2.5-mm-thick aluminum vessel that keeps the inside pressure at $1020 \mathrm{mb}$ during the flights. The total material-thickness along the particle path is 17.8 $\mathrm{g} / \mathrm{cm}^{2}$, of which 8.6 in front of the central tracker, 0.4 between IDC and JET, and 0.14 in the JET chamber gas, all in $\mathrm{g} / \mathrm{cm}^{2}$.

The trigger system is designed to detect negatively charged particles ( $\bar{p}$, antihelium) efficiently while sampling the proton and helium events in an unbiased manner. This system is composed of the "T0 trigger" and the "track trigger". The T0 trigger initiates the data gathering based upon a simple coincidence of the top and bottom scintillators with thresholds set at $1 / 3$ of the pulse height from a minimum ionizing particle. The track trigger consists of two sequential processes. The first is a pattern-selection process to reject the null-track and multi-track events by using the hit-pattern of the IDC and ODC cells. If an event passes the pattern-selection, the second process performs a rough rigidity-selection by using the cell hit-pattern. Irrespective of the track trigger condition, a fraction of T0 triggers is recorded to provide an "unbiased trigger sample", which is used to determine the efficiency of the track trigger selections. The data were collected in balloon-flights performed on July 26 '93, July 31 '94, and July 25 '95, all launched from Lynn Lake, Manitoba, Canada. During each level flight, the floating altitude was $36.5 \mathrm{~km}$ (residual atmospheric depth of $5 \mathrm{~g} / \mathrm{cm}^{2}$ ). The total live time of the three flights was 84446 sec.

The following off-line selections are applied for both negative and positive curvature events. (i) One and only one track should be found in the JET chamber. (ii) The track should be fully contained in the central six-column of the JET chamber (see fig 1), where the resolution of the rigidity-measurement is optimum. This fiducial cut determines the geometrical acceptance for the present analysis of $0.35 \mathrm{~m}^{2} \mathrm{sr}$ for '93 and '94, and $0.28 \mathrm{~m}^{2} \mathrm{sr}$ for ' 95 . The following cuts are then applied to ensure the quality of the track and the timing measurement. (1) The fitted $r \phi$-track should contain at least 14 hits in the JET chamber and at least one hit in each of the upper two and lower two IDC layers. (2) The reduced chi-square of the $r \phi$-track-fitting has to be less than 3. (3) The extrapolated track should cross the fiducial region of the TOF scintillators $(|z|<49.5 \mathrm{~cm}$ for '93 and '94, $|z|<47.0 \mathrm{~cm}$ for '95). (5) The $z$-position $\left(z_{\mathrm{TOF}}\right)$ determined by the left-right time difference measured by the PMTs should match the $z$-impact point of the extrapolated track at the TOF counter within $10 \mathrm{~cm}\left(\left|z_{\mathrm{TOF}}-z\right|<10 \mathrm{~cm}\right)$.

The particle velocity, i.e., $\beta(\equiv v / c)$ is determined from the time of flight and the path-length. Upward-moving particles are completely separated from downwardmoving ones by the sign of $\beta$ due to the excellent time resolution. We reject all upward-moving (albedo) particles at this stage, and limit further analysis to the downwardmoving particles. Fig. 2 (a) and (b) show plots of $\mathrm{d} E / \mathrm{d} x$ versus $|R|$ for the top and bottom scintillators, respectively, for a sample of ' 95 data after applying all of the above mentioned cuts. In order to select helium and antihelium, we require that events must reside in the " $\mathrm{d} E / \mathrm{d} x$ bands" shown in Fig. 2 (a) and (b). These $\mathrm{d} E / \mathrm{d} x$ cuts reject most of singly-charged particle events $\left(p, \bar{p}, e^{ \pm}\right)$, while preserving a high efficiency $(95 \%)$ for helium. Fig. 3 shows plot of $1 / \beta$ versus $|R|$ for a sample of '95 data after these $\mathrm{d} E / \mathrm{d} x$ cuts, where clear lines of ${ }^{4} \mathrm{He}$ and ${ }^{3} \mathrm{He}$ are visible. We further require that antihelium as well as helium have $\beta$ and rigidity values inside the band shown in Fig. 3. The efficiency of the $\beta$-band cut is $99 \%$ for

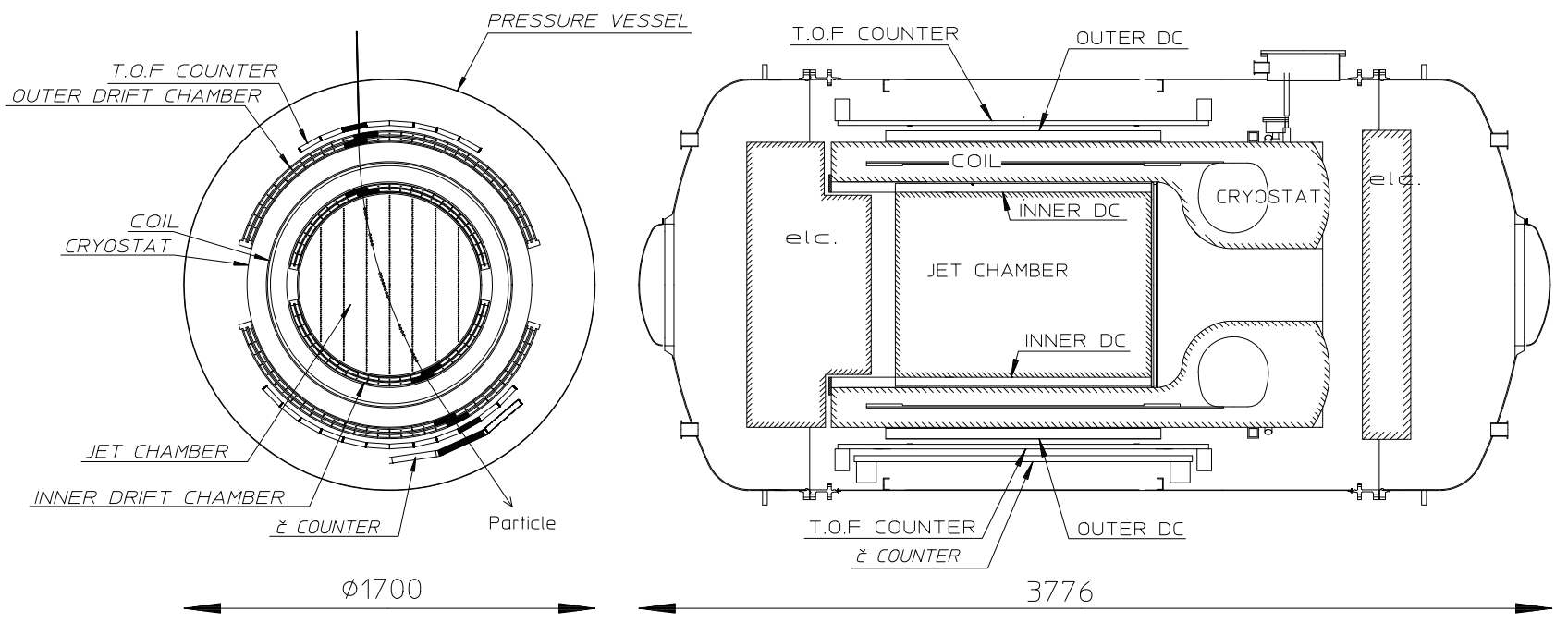

FIG. 1. Cross-sectional front- and side-views of the BESS '95 instrument. 

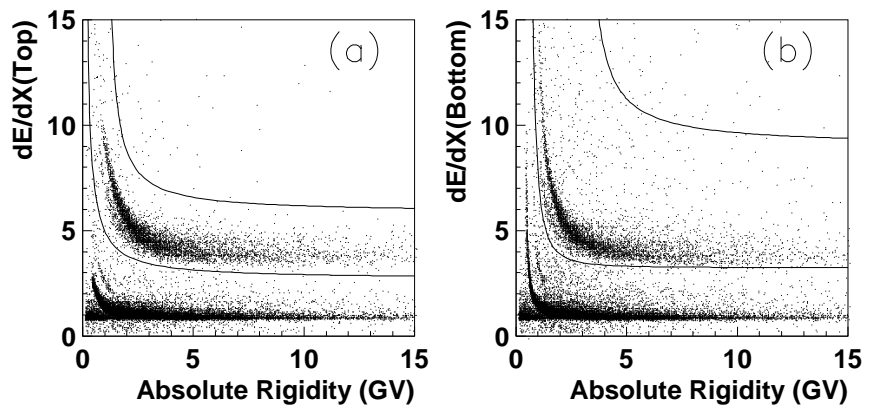

FIG. 2. Scatter plot of $\mathrm{d} E / \mathrm{d} x$ vs. absolute rigidity for (a) the top and (b) bottom layers of the TOF hodoscope ('95). The solid lines show the $\mathrm{d} E / \mathrm{d} x$ band cut positions.

helium. The overall efficiency of the off-line helium selection (from cut (1) to the $\beta$-band cut), is $63 \%, 70 \%$, and $78 \%$, for ' 93 ,' 94 , and ' 95 , respectively, and $71 \%$ in average (weighted by number of events).

All selections up to this level use $|R|$ instead of $R$, i.e. do not discriminate the positive and negative rigidity (helium and antihelium). Fig. 4 shows the $1 / R$ distribution of the events which survived all the cuts mentioned above. Those events in the negative region are apparently the spillover from the positive region due to the finite $1 / R$ resolution. There exist no events to the left of $-0.0625 \mathrm{GV}^{-1}$, which corresponds to the rigidity of -16 $\mathrm{GV}$. To define the lowest end of the rigidity region, we note that the number of helium in Fig. 4 drops sharply at $1.4 \mathrm{GV}^{-1}$, which corresponds to $1 \mathrm{GV}$ at the top of the atmosphere (TOA) after correcting for the energy losses in the instrument and in the air. Therefore, we conclude that we observed no antihelium in the rigidity region from 1 to $16 \mathrm{GV}$ at TOA.

The corresponding limit on the $\overline{\mathrm{He}} / \mathrm{He}$ flux ratio at TOA can be obtained by dividing the upper limit on the number of antihelium $\left(\overline{\mathrm{N}}_{\text {lim }}\right)$ by the total number of helium (N), which can be expressed as the integral; $\mathrm{N}=\sum_{i}\left[\mathrm{n}_{\mathrm{i}} /\left(\epsilon_{\mathrm{pat}} \epsilon_{\mathrm{sel}} \eta\right)_{\mathrm{i}}\right] / \mathrm{f}$. The summation is over the 60 bins which divide the rigidity region (1 to $16 \mathrm{GV}), \mathrm{n}_{\mathrm{i}}$ is is the number of helium detected in the unbiased trigger sample falling in the i-th rigidity bin, $\epsilon_{\mathrm{pat}}$ and $\epsilon_{\mathrm{sel}}$ are the efficiencies, for the helium, of the track-pattern- and the off-line selections, respectively, and $\eta$ is the probability of the helium surviving through the air and the instrument, all at the i-th rigidity bin. The pre-set sampling factor $\mathrm{f}$ of the unbiased trigger was $1 / 40$ for ' $93,1 / 15$ for the first half and $1 / 30$ for the last half of ' 94 , and $1 / 20$ for ' 95 . The efficiencies $\epsilon_{\mathrm{pat}}$ as well as $\epsilon_{\text {sel }}$ can be directly determined by using the unbiased trigger sample. To calculate the surviving probability $\eta$, we utilize the "inelastic" [10] cross sections of helium incident on various target nuclei, i.e. $\sigma\left(\mathrm{He}, A_{\mathrm{t}}\right)$. Since the data on $\sigma\left(\mathrm{He}, A_{\mathrm{t}}\right)$ at $\mathrm{GeV}$ energies is relatively sparse, we obtain $\sigma\left(\mathrm{He}, A_{\mathrm{t}}\right)$ by starting with $\sigma\left(p, A_{\mathrm{t}}\right)$, given as a function of incident energy [11], and by scaling it using "the hard sphere with overlap"

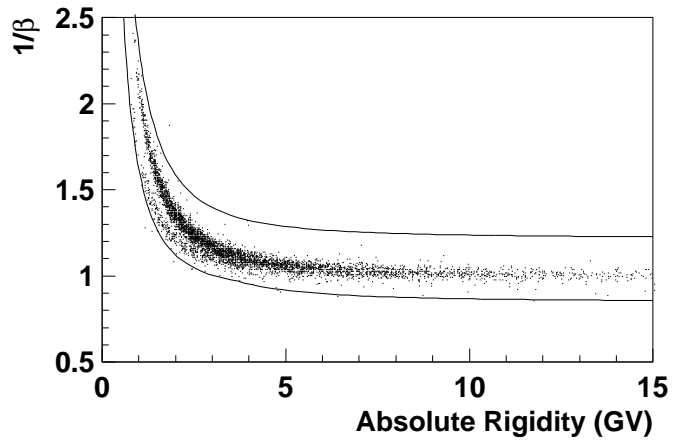

FIG. 3. Scatter plot of $1 / \beta$ vs. absolute rigidity. The solid lines show the cut positions. ('95)

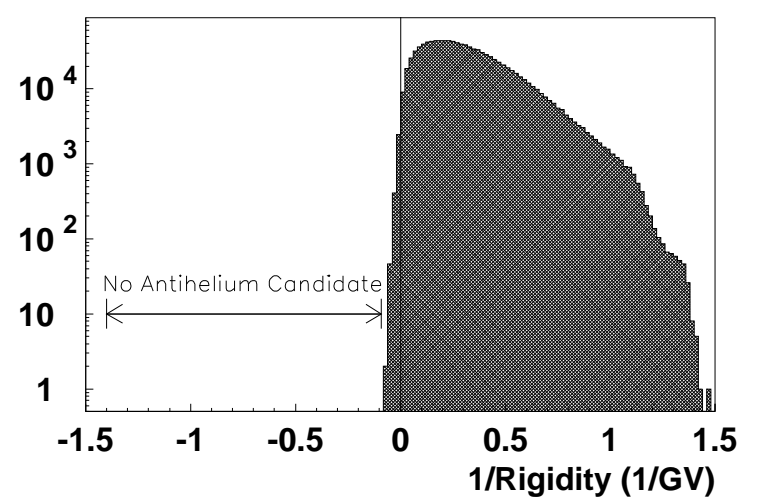

FIG. 4. 1/rigidity distribution of the events which survive all selections.

model of nuclear interaction [12], i.e.

$\sigma\left(A_{\mathrm{i}}, A_{\mathrm{t}}\right) \propto\left(A_{\mathrm{i}}^{1 / 3}+A_{\mathrm{t}}^{1 / 3}-0.71 \times\left(A_{\mathrm{i}}^{-1 / 3}+A_{\mathrm{t}}^{-1 / 3}\right)\right)^{2}$

where $A_{\mathrm{i}}$ and $A_{\mathrm{t}}$ are the atomic weight of incident and target nuclei. This model is known to reproduce data on nuclear interactions for various combinations of $A_{\mathrm{i}}$ and $A_{\mathrm{t}}$ including the proton [13]. The resultant $\sigma(\mathrm{He}$, $A_{\mathrm{t}}$ ) agrees (within an accuracy of $10 \%$ ) with measured helium cross sections on targets such as $\mathrm{C}$ or $\mathrm{Al}$. This also justifies our use of the same formula (1) to estimate the unmeasured $\sigma\left(\overline{\mathrm{He}}, A_{\mathrm{t}}\right)$ cross sections from the $\sigma(\bar{p}$, $A_{\mathrm{t}}$ ) data [14].

When summed over the rigidity bins, the number of detected helium events divided by the sampling factors $\left(\sum n_{i} / \mathrm{f}\right)$ are $2.3 \times 10^{5}, 8.7 \times 10^{5}$, and $8.6 \times 10^{5}$ for ${ }^{\prime} 93$, ' 94 , and ' 95 , respectively, and the total number of helium $\mathrm{N}$ is $5.1 \times 10^{6}$.

The upper limit on the number of antihelium $(\overline{\mathrm{N}})$ depends on its unknown rigidity spectrum, since the efficiencies are functions of rigidity. In order to obtain the most conservative limit, irrespective of the antihelium spectrum, we define $\overline{\mathrm{N}}_{\text {lim }}=3.0 /\left[\bar{\epsilon}_{\text {pat }} \bar{\epsilon}_{\text {rig }} \bar{\epsilon}_{\text {sel }} \bar{\eta}\right]_{\text {min }}$, where 3.0 is the $95 \%$ confidence level limit for zeroobserved events, and $\left[\bar{\epsilon}_{\text {pat }} \bar{\epsilon}_{\text {rig }} \bar{\epsilon}_{\text {sel }} \bar{\eta}\right]_{\text {min }}$ is the minimum value of the antihelium efficiency-product in the rigidity 


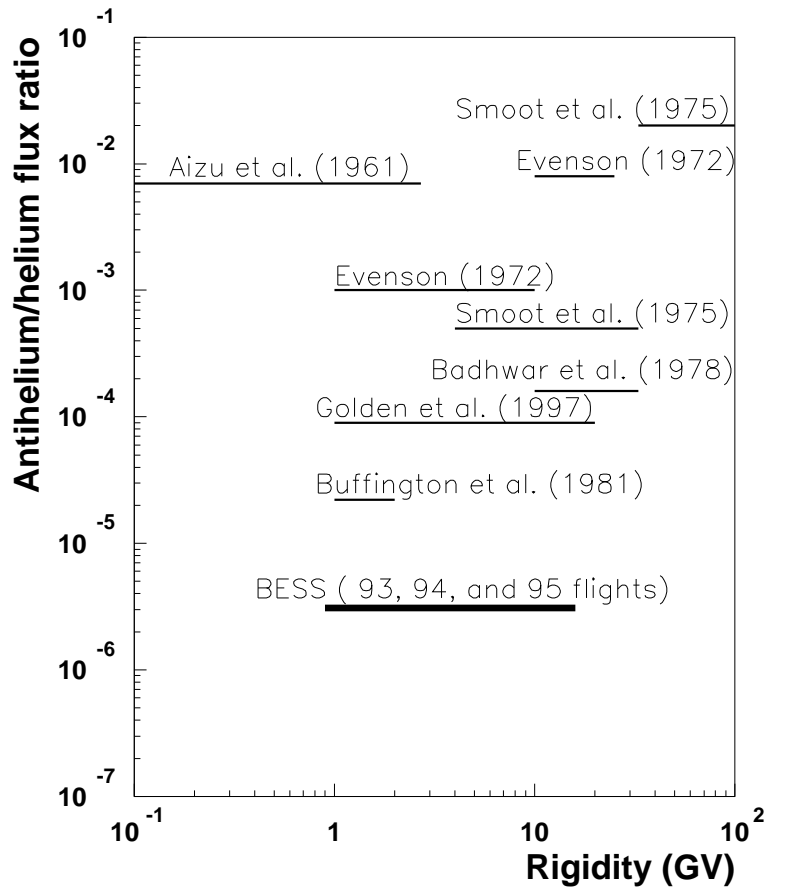

FIG. 5. The resultant upper limit of $\overline{\mathrm{He}} / \mathrm{He}$ flux ratio of the present paper together with previous limits.

region. In calculating $\left[\bar{\epsilon}_{\text {pat }} \bar{\epsilon}_{\text {rig }} \bar{\epsilon}_{\text {sel }} \bar{\eta}\right]_{\min }$, we take $\bar{\epsilon}_{\text {sel }}=\epsilon_{\text {sel }}$ and $\bar{\epsilon}_{\text {pat }}=\epsilon_{\text {pat }}$ for each rigidity bin, since the patternselection in the track trigger as well as the off-line selection does not depend on the sign of the track rigidity. The efficiency of the rigidity-selection in the track trigger $\bar{\epsilon}_{\text {rig }}$ can be directly obtained by using events with a negative rigidity track in the unbiased trigger sample, and is a monotonic function of the rigidity, having values of 0.95 at $1 \mathrm{GV}$ and 0.85 at $16 \mathrm{GV}$. The efficiency product $\left(\bar{\epsilon}_{\text {pat }} \bar{\epsilon}_{\text {rig }} \bar{\epsilon}_{\text {sel }} \bar{\eta}\right)$ thus does not vary significantly $(0.19$ at $0.7 \mathrm{GV}, 0.23$ at $2 \mathrm{GV}$, and 0.20 at $16 \mathrm{GV})$. We take the minimum value of 0.19 to calculate $\overline{\mathrm{N}}_{\text {lim }}$.

The resultant $95 \%$ C.L. upper limit on the $\overline{\mathrm{He}} / \mathrm{He}$ flux ratio at TOA is $3.1 \times 10^{-6}$ in the rigidity region from 1 to $16 \mathrm{GV}$. The upper limit on the antihelium flux integrated over the rigidity region is $6 \times 10^{-4} \mathrm{~m}^{-2} \mathrm{sr}^{-1} \mathrm{~s}^{-1}$. It should be noted that these upper limits are very conservative and are valid for any hypothetical antihelium spectrum. Our limit on the flux ratio obtained by this work is shown in Fig. 5 together with the previous ones $[2,15]$. Our flux ratio is a factor 7 improvement over the limit of Buffington et al. who looked for the annihilation signal of low rigidity $(1 \sim 2 \mathrm{GV})$ antihelium in a spark chamber calorimeter, and is 30 times more stringent than the limit of Golden et al. who covered a rigidity region similar to ours by using a previous generation magnetic rigidity spectrometer. The large acceptance of BESS, which is designed [16] specially for the antimatter and antiproton searches, made it possible to set this limit.

Authors thank NASA/GSFC/WFF Balloon office and NSBF for the balloon expeditions, and KEK and ISAS for various supports. Sincere thanks are given to Y. Ajima,
Y. Higashi and D. Righter for their help with the flights and recovery, and to A. Moiseev for discussions. This experiment was supported by NASA in the USA, and by Grant-in-Aid for Scientific Research and for International Scientific Research from the Ministry of Education, by the Kurata Research Grant, and by Sumitomo Research Grant in Japan. The analysis was performed on the RS/6000 workstations supplied for the partnership program between ICEPP and IBM Japan, Ltd.

[1] R. W. Brown and F. W. Stecker, Phys. Rev. Lett. 43, 315 (1979); K. Sato, Phys. Lett. B 99, 66 (1981).

[2] P. Evenson, Astrophys. J. 176, 797 (1972).

[3] E. Witten, Nucl. Phys. B249, 557 (1985).

[4] J. F. Ormes et al., Astrophys. J. 482, L187 (1997).

[5] R. Streitmatter, Nuovo Cimento C 19, 835; G. Tarle et al. Proceedings of ICRC 1997 (Durban) Vol. 4, 205; A. Condino and M. Lanfranchi, Astrophys. J. 487, 218C (1997).

[6] T. Saeki, Ph.D. thesis, University of Tokyo, 1996.

[7] H. Drumm et al., Nucl. Instrum. Methods 176, 333 (1980).

[8] All resolutions quoted in this paper are r.m.s. resolutions.

[9] Y. Makida et al., IEEE Trans. Appl. Supercond. 5, 658 (1995); K. Anraku et al., Adv. Space Res. 17 No.9, 101, (1996).

[10] Also denoted as "absorption" in some literatures.

[11] J. R. Letaw, R. Silberberg and C. H. Tsao, Astrophys. J. Suppl. Ser. 51, 271 (1983).

[12] H. L. Bradt, B. Peters, Phys. Rev. 77, 54 (1950); 80, 943 (1950); T. F. Cleghorn, P. S. Freier, C. J. Waddington, Can. J. Phys. 46, 572 (1968).

[13] E. O. Abdrahmanov et al., Z. Physik C 5, 1 (1980); N. S. Grigalashvili et al., Yad. Fiz. 48, 476 (1988).

[14] V. F. Kuzichev, Yu. B. Lepikhin and V. A. Smirnitsky, Nucl. Phys. A576, 581 (1994); V. F. Afonasiev et al., Yad. Fiz. 47, 1656 (1988); K. Nakamura et al., Phys. Rev. Lett. 52731 (1984); V. F. Afonasiev et al., Yad. Fiz. 40, 34 (1984); A. S. Carrol et al., Phys. Lett. B 80, 319 (1973); S. P. Denisov et al., Nucl. Phys. B 61, 62 (1973); J. C. Allaby et al., Yad. Fiz. 12, 538 (1970).

[15] R. L. Golden et al., Astrophys. J. 479992 (1997); A. Buffington et al., Astrophys. J. 248, 1179 (1981); G. D. Badhwar, R. L. Golden et al., Nature 274, 137 (1978); G. F. Smoot, A. Buffington, and C. D. Orth, Phys. Rev. Lett. 35, 258 (1975); H. Aizu et al., Phys. Rev. 121, 1206 (1961).

[16] S. Orito, Proceedings of the ASTROMAG Workshop, KEK report 87-19, 111 (1987), ed. J. Nishimura, K. Nakamura, and A. Yamamoto. 